\title{
The voiding dysfunctions in patients with non-Hunner-type interstitial cystitis/bladder pain syndrome do not affect long-term treatment outcome
}

\author{
Wan-Ru Yu${ }^{1}$, Wei-Chuan Chang ${ }^{1}$, and Hann-Chorng Kuo ${ }^{2}$ \\ ${ }^{1}$ Hualien Tzu Chi Hospital Buddhist Tzu Chi Medical Foundation \\ ${ }^{2}$ Hualien Tzu Chi Hospital, Buddhist Tzu Chi Medical Foundation, and Tzu Chi University
}

March 31, 2021

\begin{abstract}
Aims: The role of urodynamic studies in the diagnosis and prognosis of interstitial cystitis/bladder pain syndrome (IC/BPS) remains controversial. We evaluated the correlation of baseline voiding dysfunctions with long-term treatment outcome in a large cohort of patients with IC/BPS. Methods: We studied 211 patients with nonulcerative IC/BPS. All patients underwent video urodynamic examination at baseline to identify their voiding conditions and they received subsequent treatments. The primary endpoint was the global response assessment (GRA) at the current interview. Secondary endpoints included O'LearySant score (OSS), Visual Analog Scale (VAS) for pain, and the rate of IC symptom flare-up. Results: Mean patient age was $56.8 \pm 12.8$ years and mean IC symptom duration was $16.0 \pm 9.9$ years. At baseline, 83 (39.3\%) patients had a voiding problem and $62.7 \%$ had one to three comorbidities. The duration, comorbidity, treatments, changes in OSS and VAS, maximum bladder capacity (MBC), glomerulations, GRA, and flare-up rate were not significantly different among the different voiding subtypes. When we divided the patients by their voiding conditions of normal $(\mathrm{n}=32)$ and hypersensitive bladder with $(\mathrm{n}=76)$ and without $(\mathrm{n}=103)$ voiding dysfunctions, only $\mathrm{MBC}(\mathrm{P}=0.002)$ and glomerulation $(\mathrm{P}=0.021)$ demonstrated a significant difference. When we analyzed subgroups by GRA, patients with a GRA [?] 2 had a significantly shorter disease duration. There also were significant associations between GRA and the changes in OSS and VAS $(\mathrm{P}<0.001)$. Conclusions: Voiding dysfunctions in patients with non-Hunner IC/BPS do not affect long-term treatment outcome.
\end{abstract}

\section{Hosted file}

Abstract.pdf available at https://authorea.com/users/405128/articles/516159-the-voidingdysfunctions-in-patients-with-non-hunner-type-interstitial-cystitis-bladder-painsyndrome-do-not-affect-long-term-treatment-outcome

\section{Hosted file}

CM_main The voiding dysfunctions in patients with IC_BPS_WANRAT-2_MI-1.pdf available at https://authorea.com/users/405128/articles/516159-the-voiding-dysfunctions-in-patientswith-non-hunner-type-interstitial-cystitis-bladder-pain-syndrome-do-not-affect-longterm-treatment-outcome

\section{Hosted file}

Table 1.pdf available at https://authorea.com/users/405128/articles/516159-the-voidingdysfunctions-in-patients-with-non-hunner-type-interstitial-cystitis-bladder-painsyndrome-do-not-affect-long-term-treatment-outcome

\section{Hosted file}


Table 2.pdf available at https://authorea.com/users/405128/articles/516159-the-voidingdysfunctions-in-patients-with-non-hunner-type-interstitial-cystitis-bladder-painsyndrome-do-not-affect-long-term-treatment-outcome

\section{Hosted file}

Table 3.pdf available at https://authorea.com/users/405128/articles/516159-the-voidingdysfunctions-in-patients-with-non-hunner-type-interstitial-cystitis-bladder-painsyndrome-do-not-affect-long-term-treatment-outcome 